\title{
ARTICLE \\ The aryl hydrocarbon receptor regulates expression of mucosal trafficking receptor GPR15
}

\author{
Gayathri Swaminathan ${ }^{1}$, Linh P. Nguyen ${ }^{1}$, Hong Namkoong ${ }^{1}$, Junliang Pan ${ }^{2}$, Yeneneh Haileselassie ${ }^{1}$, Akshar Patel ${ }^{1}$, Allison R. Ji ${ }^{1}$, \\ David M. Mikhail ${ }^{1}$, Theresa Thanh Dinh ${ }^{2,3}$, Harpriya Singh ${ }^{1}$, Bryce Liao ${ }^{1}$, Luis M. Vázquez-Montesino ${ }^{1}$, Eugene C. Butcher ${ }^{2,3}$ and \\ Aida Habtezion (D) ${ }^{1}$
}

\begin{abstract}
GPR15 is a chemoattractant receptor that facilitates colon homing of regulatory and effector CD4 ${ }^{+} \mathrm{T}$ cells in health and colitis. The molecular mechanisms that control GPR15 expression are not fully known. Here we report the presence of two highly conserved aryl hydrocarbon receptor (AHR) binding sequences in a $3^{\prime}$ enhancer of GPR15, leading us to investigate AHR function in regulating GPR15 expression. Using luciferase reporter assays, we show that AHR activation increased GPR15 expression and requires both the AHR binding sites. Consistent with a transcriptional regulatory role, treatment with AHR agonists induce GPR15 expression on human $\mathrm{CD}^{+}{ }^{+} \mathrm{T}$ cells. Using AHR-deficient mice, we demonstrate that the lack of AHR signaling drastically reduces GPR15 expression on effector/memory and Foxp $3^{+} \mathrm{CD}^{+} \mathrm{T}$ cells. In mixed bone marrow chimeras of AHR-deficient and wildtype cells, GPR15 expression was similarly diminished on AHR-deficient $\mathrm{CD}^{+}{ }^{+}$effector/memory and regulatory $\mathrm{T}$ cells in the colon and small intestine. Furthermore, administration of AHR agonists upregulated GPR15 expression on CD4 ${ }^{+}$effector/memory T cells and increased their homing capability, especially to the colon. Collectively, our studies reveal a novel function of the AHR in regulation of GPR15 expression and increased colon trafficking of $\mathrm{CD}^{+}{ }^{+}$T cells expressing GPR15.
\end{abstract}

Mucosal Immunology (2021) 14:852-861; https://doi.org/10.1038/s41385-021-00390-x

\section{INTRODUCTION}

The G protein coupled receptor 15 (GPR15) is a lymphocyte trafficking receptor that also serves as a co-receptor for simian and human immunodeficiency virus. GPR15 is expressed on regulatory and effector/memory $\mathrm{CD}^{+} \mathrm{T}$ cells, fetal thymic dendritic epidermal T cells, B cells, and plasmablasts. This receptor mediates the colon-specific trafficking of regulatory and effector/memory $\mathrm{T}$ cells and plays a role in experimental colitis. ${ }^{1,2}$ We and others have recently identified a chemoattractant ligand of GPR15, named GPR15 ligand (GPR15L) $)^{3,4}$. This $9 \mathrm{kDa}$ polypeptide is encoded by the human gene C10orf99 and mouse gene 2610528A11Rik. It contains bridged Cysteine residues and a GPCR-activating terminal peptide, similar to classic chemokines. GPR15L induces T cell migration in a GPR15-dependent manner ${ }^{4}$. Despite these advances, the regulation of GPR15 expression is not well understood. Poly(l:C), the synthetic ligand of Toll-Like Receptor 3, induces GPR15 expression on human blood CD4 ${ }^{+}$ T cells. ${ }^{5}$ In addition, GPR15 expression is also promoted by TGF$\beta^{1,6}$ a cytokine that is abundantly expressed by intestinal epithelial cells and select gut immune cells including dendritic cells. The expression of TGF- $\beta$ itself depends on the intestinal microbiota, as TGF- $\beta$ is downregulated in germ-free mice ${ }^{7}$. In contrast, the microbiota is neither critical for the expression of
GPR15L or GPR15, ${ }^{4}$ indicating that additional signals control GPR15 expression.

To better understand the regulation of GPR15 expression, we probed a non-coding enhancer region that is downstream of the Gpr15 gene for transcription factor binding sites. We have previously demonstrated that this enhancer contains conserved regulatory elements between species and is located in a transcriptional histone active region of cells that express GPR15. ${ }^{2}$ In this enhancer, we now identify two conserved DNA binding sequences of the aryl hydrocarbon receptor (AHR) known as xenobiotic responsive elements (XREs), or dioxin responsive elements, leading us to interrogate the role of the AHR in regulating GPR15 expression.

The AHR is a cytosolic transcription factor of the PER-ARNT-SIM (PAS) protein family, whose members serve as sensors of the chemical environment. ${ }^{8}$ The AHR has long been known for its role in mediating the toxic responses to halogenated aromatic hydrocarbons, most notably the environmental pollutant $2,3,7,8-$ tetrachlorodibenzo-p-dioxin ("dioxin" or TCDD). In its inactive form, the AHR resides in a cytosolic complex of chaperone proteins made up of ARA9, p23, and two molecules of hsp90. Ligand binding triggers translocation of the receptor complex into the nucleus, where AHR is released, allowing it to heterodimerize

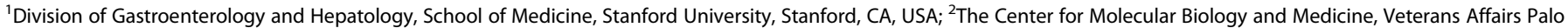

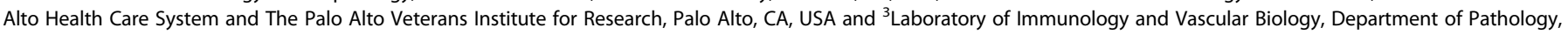
School of Medicine, Stanford University, Stanford, CA, USA

Correspondence: Gayathri Swaminathan (gswamina@stanford.edu) or Aida Habtezion (aidah@stanford.edu) These authors contributed equally: Gayathri Swaminathan, Linh P. Nguyen, Hong Namkoong, Junliang Pan Co-first Author: Junliang Pan

Co-senior Author: Eugene C. Butcher

Received: 26 August 2019 Revised: 3 February 2021 Accepted: 9 February 2021 Published online: 5 March 2021 


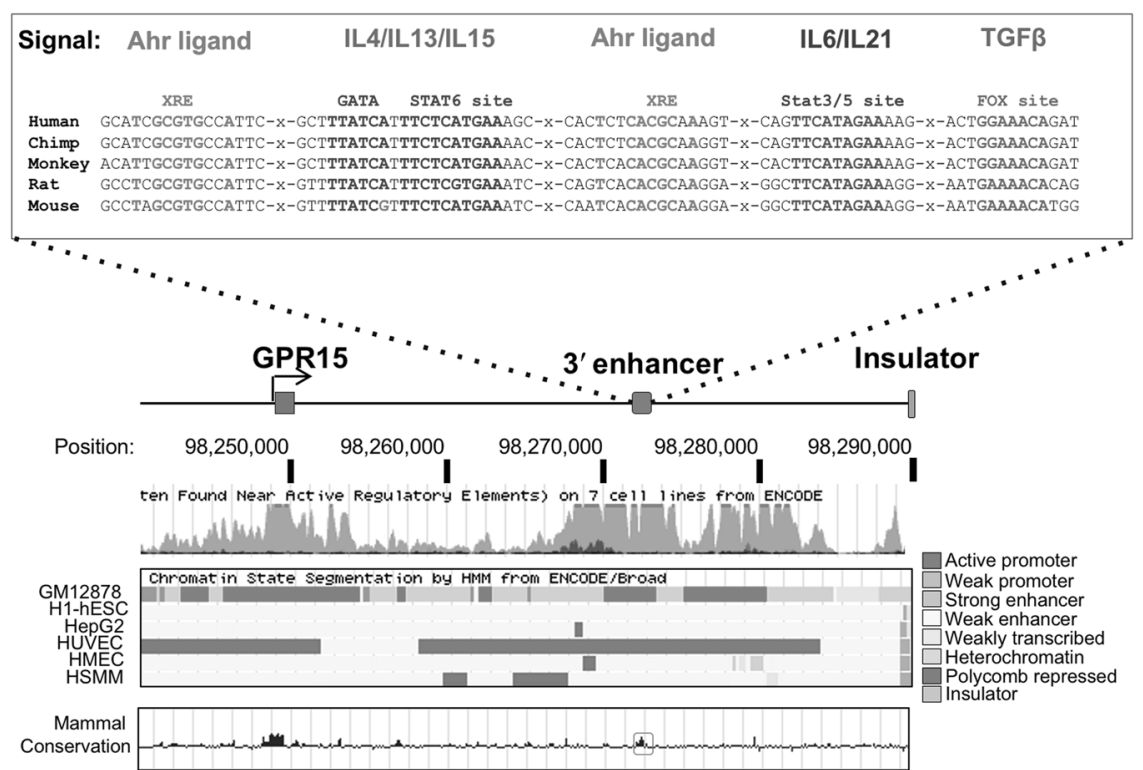

Fig. 1 Putative GPR15 $3^{\prime}$ enhancer region contains two evolutionarily conserved aryl hydrocarbon receptor (AHR)-binding sites. The GPR15 $3^{\prime}$ enhancer of $430 \mathrm{bp}$ is resident in a transcriptional active chromatin region in the GPR15-expressing lymphoblastoid cell line, GM12878, but not in GPR15-non-expressing cells such as human embryonic stem cells (H1-hESC), hepatocellular carcinoma (HepG2), human umbilical vein endothelial cells (HUVEC), human mammary epithelial cells (HMEC), and skeletal muscle myoblasts (HSMM). The indicated chromatin state segmentation in these cell types is generated by ENCODE/ Broad Institute based on Chip-seq data using specific histone markers. The $3^{\prime}$ enhancer contains a cluster of indicated transcription factor binding sites that are evolutionarily conserved and are capable of integrating potential GPR15-inducing cellular signals. The xenobiotic responsive elements (XRE) for binding to AHR are highlighted with core bases in red.

with the aryl hydrocarbon nuclear translocator (ARNT). The AHR-ARNT unit recognizes and docks on XREs that are typically located in the promoter and enhancer of transcriptionally responsive genes. ${ }^{9}$ In addition to the well-characterized AHR ligands such as polycyclic aromatic hydrocarbons (PAHs), and halogenated hydrocarbons (HAHs), a broad range of dietary, commensal, and endogenous ligands can activate the AHR. ${ }^{10-12}$ Studies in recent years have established a prominent role of the AHR in regulating mucosal immune homeostasis through integrating chemical signals of the external and internal environment, including AHR agonists derived from gut bacterial and dietary sources. Ligand-activated AHR signaling can alter innate and adaptive immune responses, including modulating $T$ cell differentiation, promoting the maturation of innate lymphoid cells, and controlling B cell class switching. ${ }^{13-15}$ The AHR also serves as the main transcriptional regulator of IL-22, a cytokine that functions in wound healing, antimicrobial defense, and intestinal epithelial integrity. ${ }^{14}$ Thus, the importance of AHR biology reaches beyond its classic toxicological and pharmacological signaling to the immune responses that are central to maintaining mucosal health. ${ }^{16-18}$

In this report, we demonstrate the importance of the AHR in regulating GPR15 expression in both mouse and human cells. We show that the GPR15 $3^{\prime}$ enhancer region contains two conserved XREs and that in vitro AHR-mediated GPR15 expression requires both functional XRE sequences in the GPR15 enhancer region. Ligand-induced AHR activation leads to GPR15 expression on colon $\mathrm{CD}^{+}{ }^{+} \mathrm{T}$ cells in vivo and on human $\mathrm{CD} 4^{+}$ $T$ cells in vitro. The absence of the AHR critically affects GPR15 expression on intestinal $\mathrm{CD} 4^{+} \mathrm{T}$ cells. Our studies thus uncover a new immune function of the AHR that affects tissue-specific homing of $T$ cells by regulating the expression of the colon trafficking receptor, GPR15. Modulating the expression of GPR15 adds to the metabolic functions of the AHR, which may serve to respond to dietary antigens or exogenous pathogens through the increased colon trafficking of effector $\mathrm{CD}^{+}{ }^{+} \mathrm{T}$ cells that express GPR15.

\section{RESULTS}

Noncoding enhancer region $3^{\prime}$ of GPR15 contains AHR binding sites

To probe the mechanisms underlying the regulation of GPR15 expression, we performed a comparative sequence analysis of an enhancer region that is $3^{\prime}$ of the GPR15 gene. We have previously shown that this noncoding region harbors a cluster of transcription factor binding sites and resides in a transcriptionally active chromatin region of the GPR15-expressing lymphoblast cell line GM12878; in cell lines that do not express GPR15, this chromatin region remains inactive. ${ }^{2}$ In this enhancer, we now identify two AHR binding sites/XREs that are conserved across mammalian species. These XREs contain the invariant core consensus sequence $5^{\prime}$-GCGTG-3' (Fig. 1) and are located 135 bp apart in human and $133 \mathrm{bp}$ apart in mouse. This observation led us to hypothesize that the AHR transcriptionally regulates GPR15 expression.

\section{AHR ligand induced GPR15 expression in an XRE-dependent manner}

We first tested the importance of the AHR binding sites present in the GPR15 enhancer region on AHR-mediated GPR15 transcription using the luciferase reporter assay. Human epithelial HEK293 cells were transiently transfected with a GPR15 promoter-driven luciferase construct that contains the two XRE-bearing enhancer region sub-cloned downstream of the luciferase reporter gene (Fig. 2a). In cells transfected with the construct that contained the two intact XREs, the endogenous AHR agonists FICZ and biliverdin, the bacterial virulent factor 1-hydroxyphenazine and pyocyanin, or TCDD induced luciferase reporter activity in a dose-dependent manner while the small molecule AHR antagonist, CH223191, decreased GPR15-reporter activity (Fig. 2b, Supplementary Fig. 1). TCDD also dose-dependently induced luciferase activity in Jurkat cells that were electroporated with the wildtype construct (not shown). Reporter activity was diminished when the transfected construct contained mutations in either of the AHR binding sites, and further abrogated when mutations occurred in both XREs (Fig. 2c). Agonist-induced activity was also inhibited by the AHR 
a

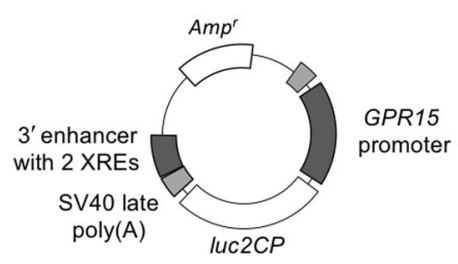

b

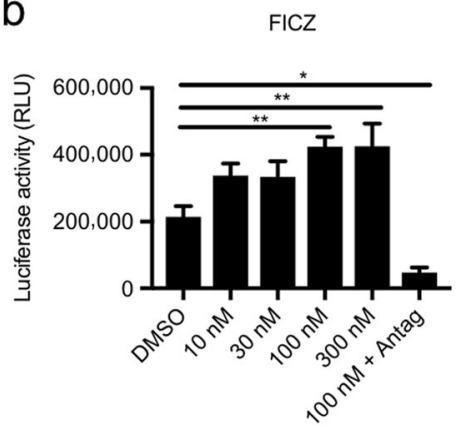

Biliverdin

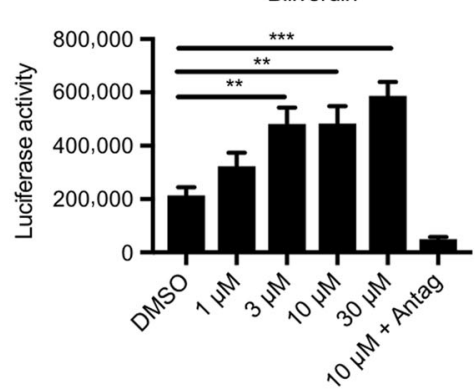

C

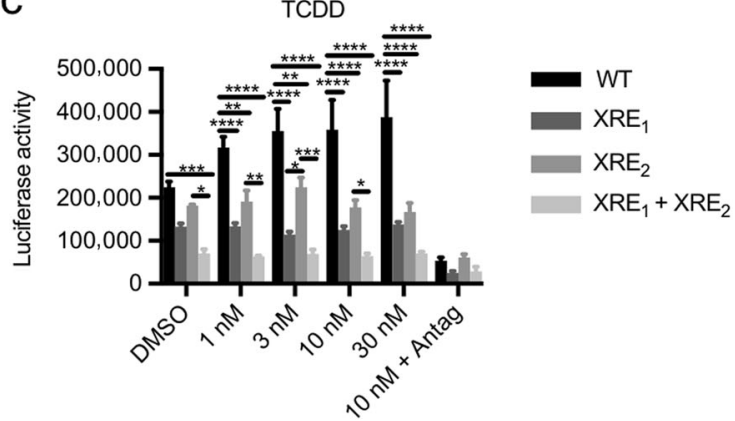

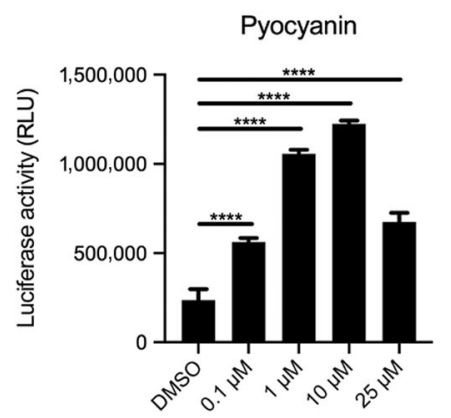

Fig. 2 AHR agonist induces luciferase reporter activity in an XRE-dependent manner. a A luciferase reporter plasmid was subcloned with $5^{\prime}$ GPR15 promoter and $3^{\prime}$ enhancer region containing two wildtype or mutant XREs. b HEK293 cells were transiently transfected with the plasmid containing the wildtype XREs and treated with indicated doses of AHR agonist 6-formylindolo[3,2-b]carbazole (FICZ), biliverdin or 1hydroxyphenazine or pyocyanin in the absence or presence of $5 \mu \mathrm{M}$ antagonist $\mathrm{CH} 223191$ (Antag). c HEK293 cells were transiently transfected

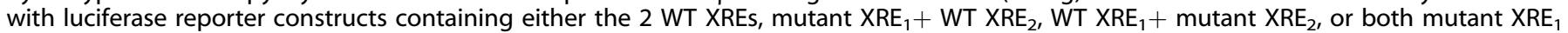
and $X_{R E}$. The transfected cells were treated with indicated doses of TCDD in the absence or presence of $5 \mu \mathrm{M}$ antagonist $\mathrm{CH} 223191$ (Antag). $n=4$, mean + SEM. One-way ANOVA with Dunnett's posttest. ${ }^{*} P<0.05,{ }^{* *} P<0.01,{ }^{* *} P<0.001,{ }^{* * *} P<0.0001$. Data representative of $2-3$ experiments.

antagonist, $\mathrm{CH} 223191$. These studies indicate that GPR15 expression can be upregulated by the AHR agonists FICZ and biliverdin, 1-hydroxyphenazine, pyocyanin, as well as TCDD. Importantly, the XREs in the GPR15 $3^{\prime}$ enhancer region is critical for the AHRmediated GPR15 expression.

AHR agonists induce GPR15 expression on $\mathrm{CD}^{+} \mathrm{T}$ cells of human PBMC

We next tested the effect of AHR modulation on GPR15 expression in human PBMC. The treatment of activated human PBMC from healthy donor with the AHR agonists TCDD or FICZ increased GPR15 gene expression, consistent with a transcriptional regulatory role of AHR on GPR15 expression. Conversely, treatment with AHR antagonist, $\mathrm{CH} 223191$, decreased GPR15 expression (Supplementary Fig. 2a), similar to levels as the well-studied AHR target genes, Cyp1a1/ Cyp1b1 (data not shown). Several immune cell subsets in human PBMC express GPR15 and AHR. To test if the AHR mediates increase of GPR15 in CD4 ${ }^{+} \mathrm{T}$ cells, we analyzed the effect of two recently discovered AHR agonists derived from bacteria, pyocyanin or 1-hydroxy phenazine on GPR15 expression by FACS analysis. AHR agonist treatment induced GPR15 expression in stimulated human $\mathrm{CD}^{+} \mathrm{CD}^{+} 5 \mathrm{RO}^{+} \mathrm{T}$ cells (Fig. 3, Supplementary Fig. 2b), while antagonist treatment decreased GPR15 expression (Supplementary Fig. 2c) supporting the hypothesis that the AHR regulates GPR15 expression. Furthermore, in vitro treatment of $\mathrm{CD}^{+}{ }^{+} \mathrm{CD} 45 \mathrm{RO}^{+} \mathrm{T}$ cells sorted from human PBMC with the AHR agonist, pyocyanin increased GPR15 expression suggesting a direct effect of $\mathrm{AHR}$ on $\mathrm{CD}^{+}{ }^{+} \mathrm{T}$ cells and consistent with our PBMC results. GPR15 expression in sorted and cultured $\mathrm{CD}^{2} 5 \mathrm{RO}^{-} \mathrm{CD}^{+} \mathrm{T}$ cells was negligible $(\sim 1 \%)$ when compared to CD45RO ${ }^{+}$T cells ( $30 \%$ ) (Fig. $3 \mathrm{~b}$ and Supplementary Fig. 3).

AHR-mediated human GPR15 expression is modulated by GATA3 and Foxp3

We had previously reported that GATA3 and Foxp3 can bind to human GPR15 $3^{\prime}$ enhancer and regulate its expression on $\mathrm{CD}^{+}$ T cells. ${ }^{2}$ In light of our current finding that AHR facilitates GPR15 expression and prompted by reports of AHR interactions with transcription factors such as Foxp3 in mediating GPR15 expression, ${ }^{19}$ we next delineated the contribution of AHR, GATA3, and Foxp3 in human GPR15 expression. We determined how 
a

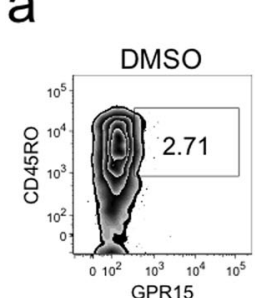

1-Hydroxy phenazine

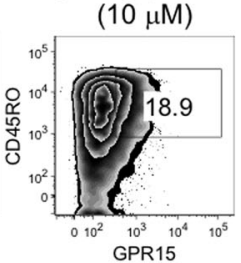

Pyocyanin

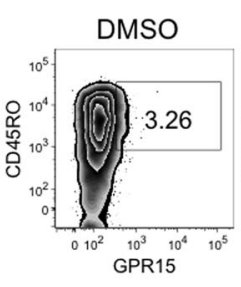

Pyocyanin
$10 \mu \mathrm{M}$

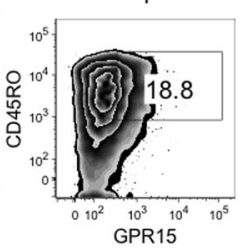

b

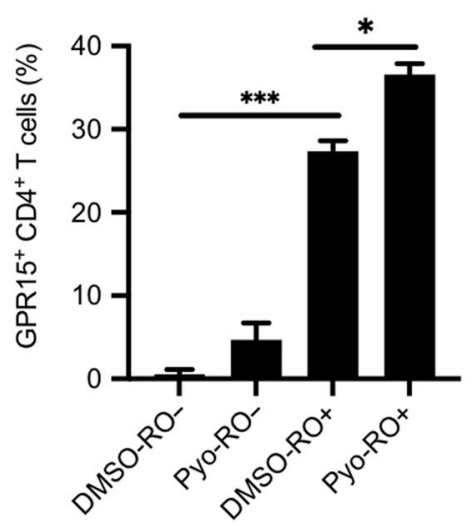

1-Hydroxy phenazine Pyocyanin

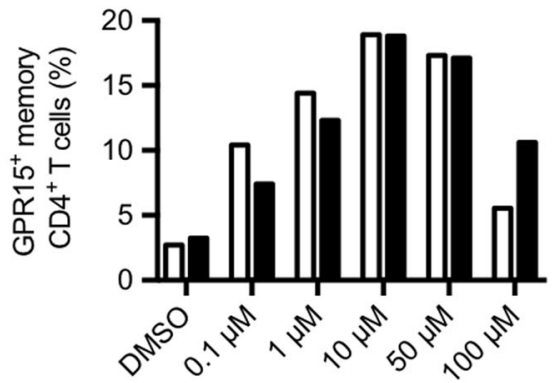

Fig. 3 AHR agonist induced GPR15 expression on human blood CD4 ${ }^{+}$T cells. a Activated peripheral blood mononuclear cells (PBMC) from healthy donor were treated with AHR agonists as indicated for $24 \mathrm{~h}$ and assessed by flow cytometry for expression of GPR15 on memory $\mathrm{CD}_{5} \mathrm{RO}^{+} \mathrm{CD}^{+} \mathrm{T}^{\mathrm{T}}$ cells. $\mathbf{b} \mathrm{CD} 45 \mathrm{RO}^{+}$and $\mathrm{CD} 45 \mathrm{RO}^{-} \mathrm{CD}^{+} \mathrm{T}$ cells were sorted from peripheral blood mononuclear cells of healthy donors and treated with AHR agonist, pyocyanin (Pyo; $10 \mu \mathrm{M}$ ) or DMSO as indicated for $48 \mathrm{~h}$ and assessed by flow cytometry for expression of GPR15. Mean + SEM. One-way ANOVA and Tukey's posttest, ${ }^{*} P<0.05,{ }^{* *} P<0.001$.

expression of AHR, GATA3, Foxp3 alone or in combination affected GPR15-luciferase reporter expression. We observed that transfection of AHR or GATA3 increased GPR15-driven luciferase activity and that there was an additive effect when they were cotransfected (Supplementary Fig. 4). Mutation of the AHR binding sites $\left(X E_{1}\right.$ and $\left.X R E_{2}\right)$ or inhibition of AHR activity using the AHR antagonist, $\mathrm{CH} 223191$, reduced but did not abolish GATA3mediated increase in GPR15 expression suggesting independent as well as synergistic effects of GATA3 and AHR on GPR15 expression. Interestingly, transfection of Foxp3 decreased GPR15 expression in our luciferase assays (Supplementary Fig. 4). Our results validate that AHR signaling promotes GPR15 expression and provide some insights on how AHR cross-talks with GATA3 and Foxp3 to influence human GPR15 expression.

Consistent with the synergistic effects of AHR and GATA3 on GPR15 expression in the reporter assays, modulation of AHR activity in human $\mathrm{CD}^{+}{ }^{+} \mathrm{T}$ cells cultured under Th2 polarizing conditions with AHR agonists or antagonist resulted in an increase or decrease, respectively, in GPR15 expression (Supplementary Fig. 5a, c). Our results indicate that AHR signaling and GATA3 binding to the GPR15 $3^{\prime}$ enhancer may act in concert to promote GPR15 expression on human Th2 cells. In addition, treatment of in vitro polarized human Tregs with AHR agonists or antagonist showed similar responses as that observed with Th2 cells (Supplementary Fig. 5b, c). In all our experiments, we consistently noted significantly higher GPR15 expression on in vitro polarized human Th2 compared to Tregs. Depending on AHR signaling status, GPR $15^{+}$Tregs varied between $0.8 \%$ and $53 \%$ while GPR $15^{+}$ Th2 cells were in the range of $3.6-85 \%$ after $5-7$ days of culture in differentiation media with or without AHR agonists and antagonist. Our data thus affirms the role of AHR as an important regulator of GPR15 expression in human $\mathrm{CD}^{+}{ }^{+} \mathrm{T}$ cells including Th2 and Tregs.

AHR deficiency impacts GPR15 expression and intestinal T cell populations in vivo

We and others have shown that in healthy mice, GPR15 is more highly expressed on $\mathrm{CD}^{+}{ }^{-} \mathrm{T}$ cells of the colon than the small intestine or other tissues ${ }^{1,2}$ with about $20-25 \%$ of colonic lamina propria effector/memory CD4 ${ }^{+}$T cells expressing GPR15 (Fig. 4a). ${ }^{2}$ Given our observations on the in vitro effect of AHR on GPR15 expression, we further tested effects of AHR modulation on GPR15

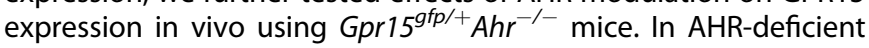
mice, GPR15 (GFP) expression on colonic lamina propria effector/ memory $\mathrm{CD}^{+}{ }^{+} \mathrm{T}$ cells (alternatively referred to as memory $\mathrm{CD} 4^{+}$ T cells) decreased by nearly $60 \%$. Expression was also significantly reduced on effector/memory $\mathrm{CD}^{+}{ }^{+} \mathrm{T}$ cells of the small intestine but was not affected in other tissues or on naive $\mathrm{CD} 4^{+} \mathrm{T}$ cells where GPR15 expression is normally minimal (Fig. 4a, b). In healthy wildtype mice, GPR15 is primarily co-expressed with CD25 on Foxp $^{+} \mathrm{CD}^{+}$regulatory T cells, and GPR15 is also expressed on effector/memory (CD44 ${ }^{\text {hi }}$ CD45RB $\left.{ }^{\text {lo }}\right) \mathrm{CD}^{+} \mathrm{T}$ cells under 

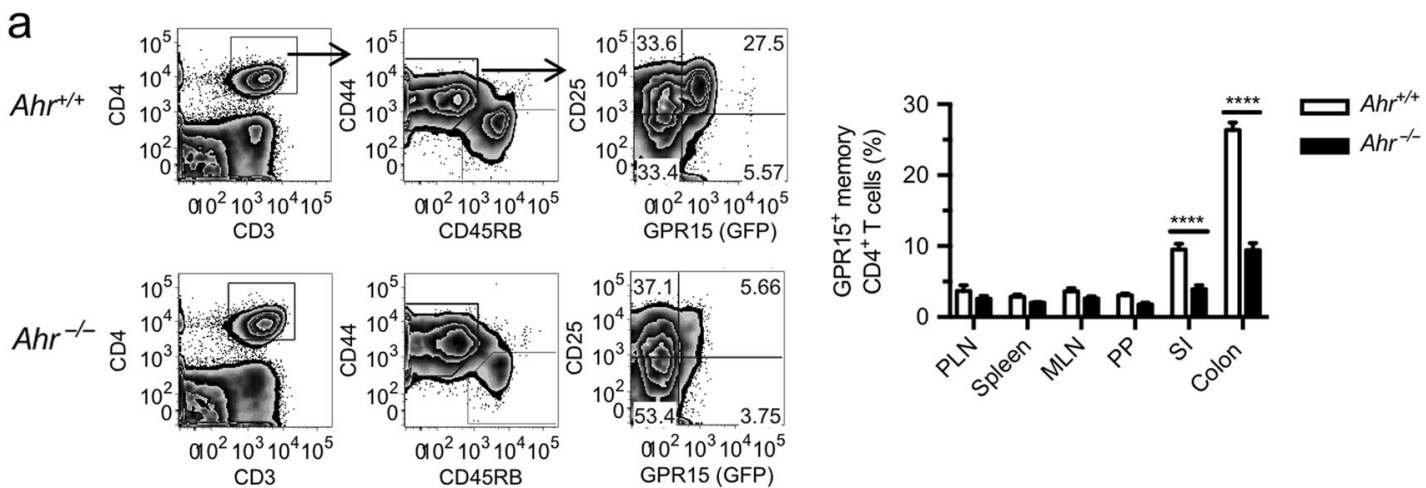

b
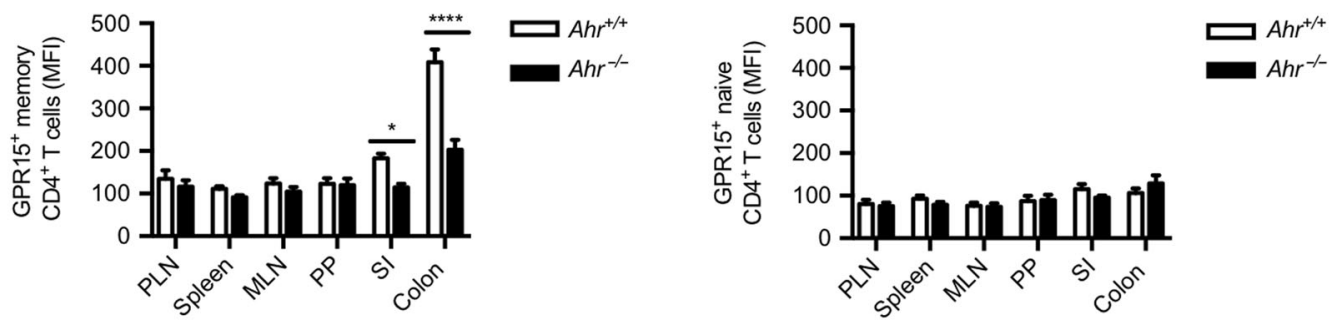

Fig. 4 AHR deficiency limits GPR15 expression on intestinal lamina propria memory CD4 ${ }^{+} \mathbf{T}_{\text {cells. a Reduced GPR15 }}^{+}$memory CD4 ${ }^{+}$ $\mathrm{T}$ cells in intestinal lamina propria of $\mathrm{Gpr} 15^{g f p /+} \mathrm{Ahr}^{-/-}$than $\mathrm{Gpr} 15^{g f p /+} \mathrm{Ahr} r^{+/+}$mice. Colon lamina propria lymphocytes as gated on memory CD44 ${ }^{\text {hi }}, \mathrm{CD}_{4} \mathrm{RB}^{\mathrm{lo}} \mathrm{CD}^{+}$T cells. b Decreased mean fluorescence intensity (MFI) of GPR15 expression by $\mathrm{Ahr}^{-/-}$memory (left) but not naive (right) $\mathrm{CD}^{+}$T cells. PLN peripheral lymph nodes, MLN mesenteric lymph nodes, PP Peyer's patches, SI small intestine. Mean + SEM, $n=$ $13 \mathrm{Ahr}^{++_{+}^{+}}$and $12 \mathrm{Ahr}^{-/-}$. Two-way ANOVA with Bonferroni posttest, ****P<0.0001. Data of three combined experiments.

inflammatory conditions ${ }^{2}$. In AHR knockout mice, GPR15 expression decreased on both $\mathrm{CD}_{2} 5^{+} \mathrm{CD} 4^{+} \mathrm{T}$ cells as well as CD25 $5^{\text {lo }}$ effector/memory $\mathrm{CD}^{+} \mathrm{T}$ cells (Supplementary Fig. 6a, b). AHR deficiency, however, did not alter the expression of CD25 itself, as the percentage of $\mathrm{CD}_{2} 5^{+} \mathrm{CD} 4^{+}$did not differ significantly between wildtype and AHR knockout mice (Supplementary Fig. 6c). Similar results were obtained using $\mathrm{Ahr}^{-/-}$mice, following staining of different tissues with an anti-GPR15 antibody and flow cytometry analysis. AHR deficiency decreased GPR15 expression on intestinal lamina propria $\mathrm{CD} 25^{+} \mathrm{Foxp}^{+} \mathrm{CD}^{+} \mathrm{T}$ cells as well as effector/memory $\mathrm{CD}^{+}{ }^{+} \mathrm{T}$ cells (Supplementary Fig. 7). On the other hand, AHR deficiency was associated with increased frequency of intestinal GPR $15^{-} \mathrm{CD}_{2} 5^{+}$Foxp $^{+}$and effector/ memory $\mathrm{CD}^{+} \mathrm{T}$ cells (Supplementary Fig. 7). These results indicate that the AHR plays a role in regulating GPR15 expression on both effector/memory as well as regulatory $C D 4^{+} \mathrm{T}$ cells. AHR deficiency, however, did not abolish GPR15 expression completely, suggesting that GPR15 regulation might also occur through an AHR-independent mechanism.

AHR impacts GPR15 expression and intestinal T cell populations in bone marrow chimeras

To further assess the importance of the AHR on GPR15 expression and GPR15-dependent gut reconstitution of $\mathrm{CD}^{+}{ }^{+} \mathrm{T}$ cells in vivo, we set up mixed bone marrow chimeras, in which congenic allotype-marked Gpr15 ${ }^{g f p /+} A \mathrm{Ar}^{-/-}$cells (CD45.1, CD45.2) were allowed to compete with $\mathrm{Gpr} 15^{g f p /+} \mathrm{Ahr}^{+/+}$cells (CD45.1) for repopulating $\mathrm{CD4}^{+} \mathrm{T}$ cell compartments of the intestinal and lymphoid tissues (Fig. 5a). After cell engraftment, fewer colon effector/memory $\mathrm{CD}^{+}{ }^{+} \mathrm{T}$ cells that derived from $\mathrm{Ahr}^{-/-}$donors expressed GPR15 than did their wildtype counterparts (Fig. 5b, c). A similar effect was also observed in the small intestine. As anticipated, naive CD4 ${ }^{+}$T cells did not express GPR15 and this did not change with AHR deficiency (Fig. 5d, e). Similar to observations with $\mathrm{Ahr}^{-1-}$ mice, AHR deficiency was associated with increase in GPR15 ${ }^{-}$effector/memory $\mathrm{CD}^{+}{ }^{+} \mathrm{T}$ cells in the small intestine (Supplementary Fig. 8). Consistent with these findings, our mixed bone marrow chimera experiments using $A h r^{+/+}$(CD45.1) and $\mathrm{Ahr}^{-1-}$ (CD45.2) donor mice and staining with anti-GPR15 antibody showed decreased GPR15 expression on $\mathrm{Ahr}^{-1-}$ derived colon lamina propria effector/memory $\mathrm{CD}^{+}{ }^{+} \mathrm{T}$ cells as well as Foxp3 $^{+} \mathrm{CD}^{+} \mathrm{T}$ cells compared to $\mathrm{Ahr}^{+/+}$derived donor cells (Supplementary Fig. 9). The results of this study are consistent with an important function of the AHR in regulating GPR15 expression on intestinal $\mathrm{CD} 4^{+} \mathrm{T}^{\mathrm{T}}$ cells.

AHR agonists induce GPR15 expression in vivo

We next assessed the effect of AHR activation on GPR15 expression in vivo by treating mice with AHR agonists. The small molecule indole-3-carbinol (I3C), commonly found in cruciferous plants, was shown to readily convert to potent AHR agonists in the acidic gastric environment. ${ }^{20}$ We therefore tested I3C because the site of their exposure could provide insights on 'local' AHRdependent regulation of GPR15 expression on $\mathrm{CD} 4^{+} \mathrm{T}$ cells and GPR15 biology. Oral treatment with I3C (at $600 \mathrm{mg} / \mathrm{kg}$ ) led to increased GPR15 expression on effector/memory CD4 ${ }^{+} \mathrm{T}$ cells in the colon and small intestine (Fig. 6a). We next tested the effect of the classical, well-characterized, high-affinity AHR ligand, TCDD on GPR15 expression in vivo. TCDD is not susceptible to metabolic breakdown and has a long half-life in vivo and induces sustained AHR activation which is maintained at least for 1-2 weeks. $^{21}$ GPR15 expression showed significant increase with systemic treatment of TCDD (Fig. 6b, c). With i.p. injection of $10 \mu \mathrm{g} / \mathrm{kg}$ TCDD, GPR15 expression increased on effector/ memory CD4 ${ }^{+}$ T cells of the colon (to over $50 \%$ ) and of tissues that normally do not express GPR15. Interestingly, a GPR15-bright effector/memory $\mathrm{CD}^{+} \mathrm{T}$ cell population that was primarily (but not strictly) CD25 $5^{\text {lo }}$ became apparent in the colon (located by an arrow, Fig. 6c). Administration of $13 C$ and $1 \mu \mathrm{g} / \mathrm{kg}$ TCDD did not significantly impact intestinal GPR15-negative effector/memory T cells while $10 \mu \mathrm{g} / \mathrm{kg}$ TCDD caused a decrease in GPR15-negative effector/ memory cells in the small intestine (Supplementary Fig. 10). In addition, the TCDD dose of $10 \mu \mathrm{g} / \mathrm{kg}$ also induced expression of integrin $a 4 \beta 7$, but not the small intestine-trafficking chemokine 
a
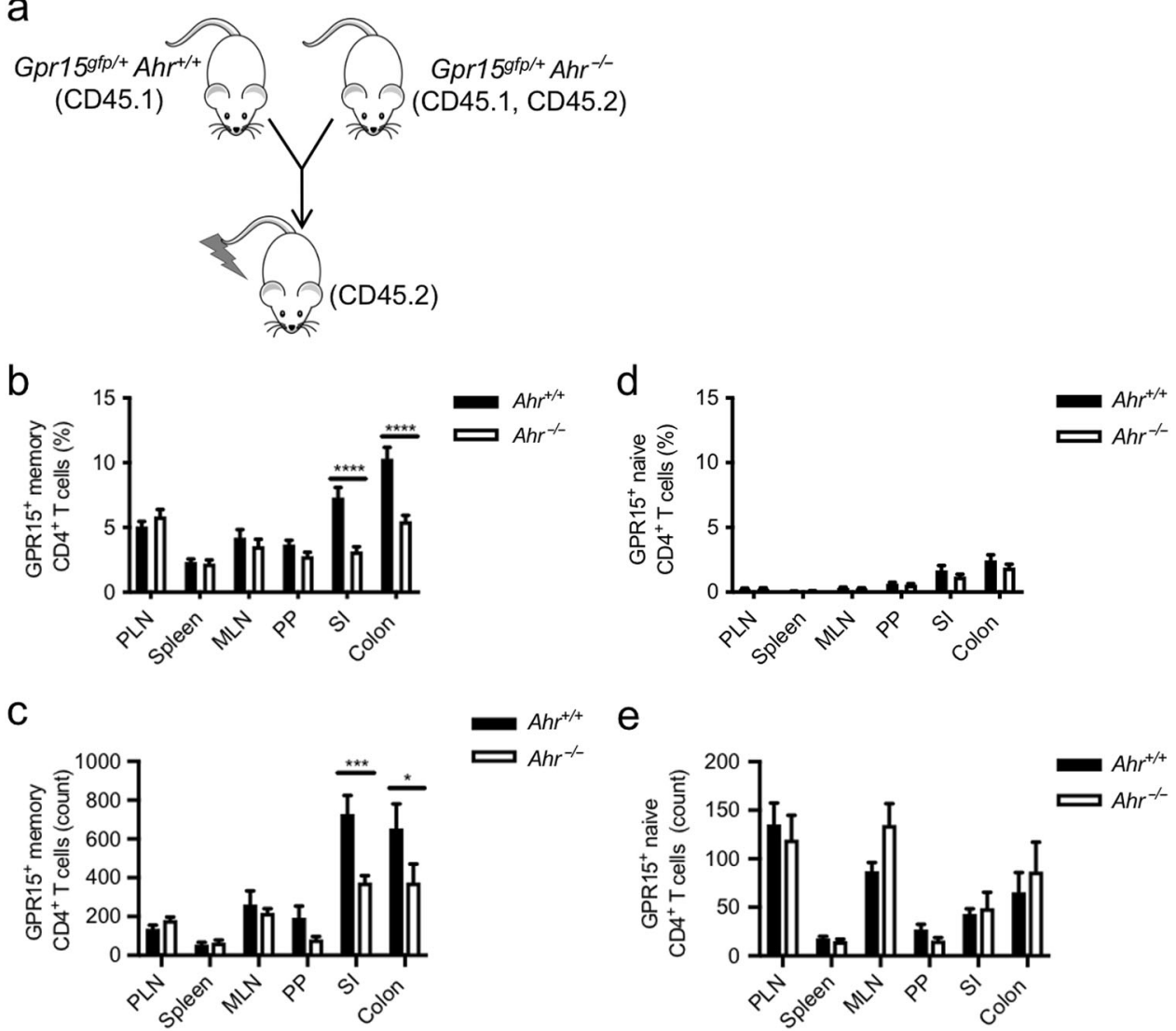

Fig. 5 AHR deficiency impacts intestinal $\mathrm{GPR}^{+} 5^{+}$memory $\mathrm{CD}^{+} \mathrm{T}$ cells in competitive bone marrow chimeras. a Bone marrow from Gpr15 $5^{g p /+} \mathrm{Ahr}^{+/+}(\mathrm{CD} 45.1)$ and Gpr15 $5^{g f p /+} \mathrm{Ahr}^{-/-}$(CD45.1, CD45.2) are engrafted in lethally irradiated normal WT hosts (CD45.2). Tissues were harvested 6-10 weeks post injection. b, c Frequency and number of donor-derived GPR15 (GFP) ${ }^{+}$memory CD44 $^{\text {hi }}$, CD45RB ${ }^{\text {lo }}$ CD4 ${ }^{+}$ $\mathrm{T}$ cells. d, e Frequency and number of naive CD44 ${ }^{\mathrm{lo}}, \mathrm{CD}_{5} \mathrm{RB}^{\mathrm{hi}} \mathrm{CD} 4^{+} \mathrm{T}$ cells of host tissues. PLN peripheral lymph nodes, MLN mesenteric lymph nodes, PP Peyer's patches, SI small intestine. Mean + SEM; $n=14$, two-way ANOVA with Bonferroni posttest, ${ }^{*} P<0.05{ }^{* * * * P<0.0001 .}$ Combined results of three independent experiments.

receptor CCR9 on colon memory $\mathrm{CD}^{+} \mathrm{T}$ cells (Supplementary Figs. 11 and 12). The TCDD-mediated increase in GPR15 expression on intestinal $\mathrm{CD}^{+}{ }^{+} \mathrm{T}$ cells required the presence of $\mathrm{AHR}$ as TCDD administration in $\mathrm{Ahr}^{+/+}$, but not $\mathrm{Ahr}^{-/-}$mice caused increased GPR15 expression on effector/ memory CD4 ${ }^{+}$ T cells and $\mathrm{CD}_{2} 5^{+} \mathrm{Foxp}^{+} \mathrm{CD} 4^{+} \mathrm{T}$ cells (Supplementary Fig. 13). Importantly, GPR15 ${ }^{+}$effector/memory CD4 ${ }^{+}$T cells isolated from TCDD-treated mice showed higher gut-homing capability compared to GPR $15^{-}$effector/memory $\mathrm{CD}^{+} \mathrm{T}$ cells in a short-term gut homing assay (Supplementary Fig. 14). Our data demonstrate the ability of diverse AHR ligand sources; I3C (dietary) and TCDD (environmental toxin) to upregulate GPR15 expression in vivo and augment the recruitment of $\mathrm{CD}^{+}{ }^{+} \mathrm{T}$ cells to the colon in a GPR15dependent manner.

\section{DISCUSSION}

We and others have previously shown that GPR15 mediates CD4 ${ }^{+}$ $T$ cell traffic to the colon and plays an important role in colitis. The identification of its chemoattractant, GPR15L, and the demonstrated expression of GPR15L in the colon have solidified the function of GPR15 as a colon-specific T cell trafficking receptor. Given the translational potential of targeting GPR15-GPR15L axis for colitis treatment, we sought to elucidate the mechanisms that regulate GPR15 expression and function. We previously identified an enhancer region that is $3^{\prime}$ of the GPR15 open reading frame. This enhancer contains DNA binding sites for GATA3 and Foxp3, the master transcription factors of the respective Th2 and Treg lineages, that regulates GPR15 expression in a species-specific manner. ${ }^{2}$ In this investigation, we report the presence of two highly conserved DNA binding sites for the AHR in the $3^{\prime}$ enhancer region of GPR15. Determining the contribution of the AHR to GPR15 expression would advance our understanding of how effector $\mathrm{CD}^{+} \mathrm{T}$ cell recruitment might be modulated for the treatment of inflammatory bowel disease. Consistent with a conserved transcriptional regulatory role, our studies reveal that modulation of AHR expression and/or signaling regulated GPR15 expression on Tregs and effector/memory cells in both mice and humans.

Our studies using a luciferase reporter gene system revealed the importance of the AHR binding sites/XREs in the GPR15 $3^{\prime}$ enhancer and AHR activity in GPR15 expression. In the presence of both wildtype XREs, reporter activity was induced dosedependently by different AHR agonists and inhibited by the antagonist $\mathrm{CH} 223191$. We observed that antagonist treatment significantly lowered reporter transcriptional activity relative to vehicle alone, indicating the presence of AHR agonists in the cell culture media. Indeed, DMEM-based cell culture media that contains high levels of aromatic amino acids, such as tryptophan, can readily generate AHR agonists. ${ }^{22}$ Importantly, we found that when either or both XRE sequences were altered, reporter activity was additively diminished, underscoring the direct involvement of the AHR binding XREs in the GPR15 $3^{\prime}$ enhancer in regulating GPR15 expression. Further interrogation of the effect of GATA3 and Foxp3 expression on AHR-mediated GPR15 reporter expression revealed that GATA3 enhances while Foxp3 reduces 

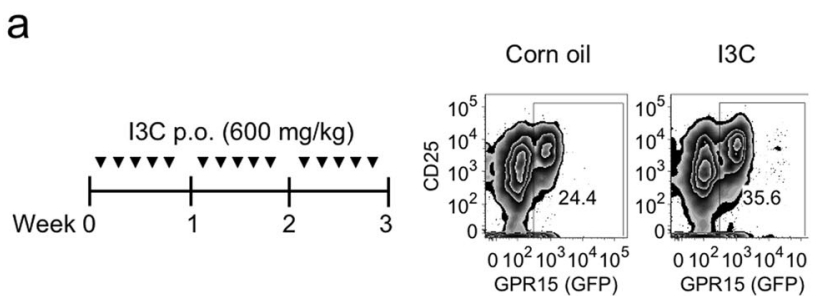

b
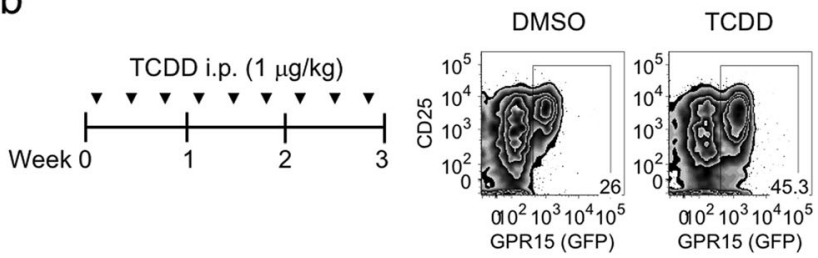

C

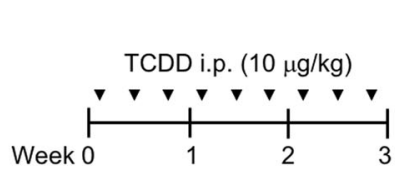

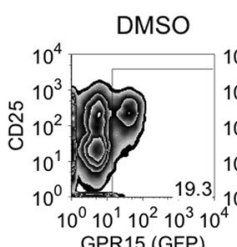

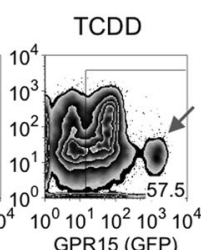

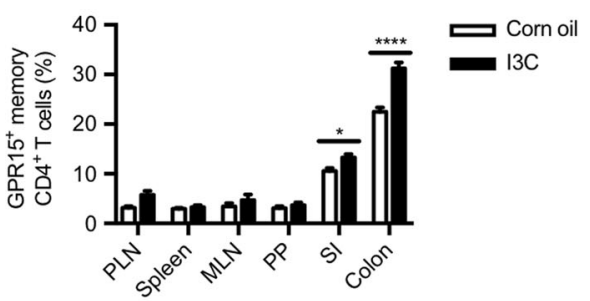
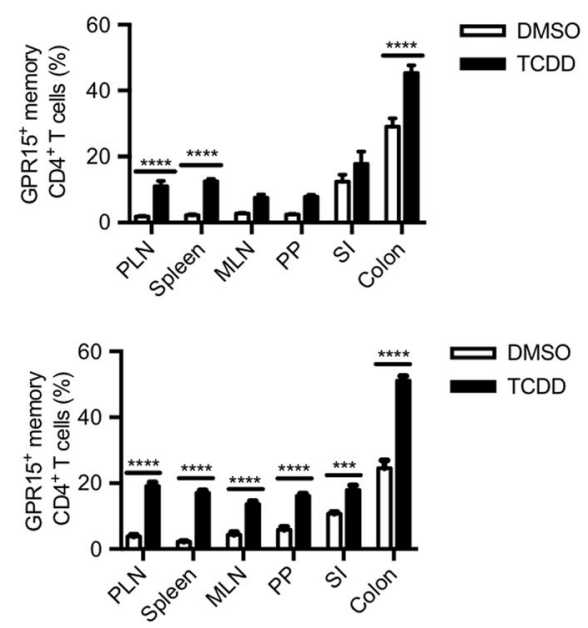

Fig. 6 AHR agonists increase GPR15 expression in vivo. Gpr $15^{g f p /+} A h r^{+/+}$were orally gavaged with indole-3-carbinol or corn oil, or i.p. injected with TCDD and DMSO as shown in the figure. Representative FACS plots of gated CD4 ${ }^{+}{\text {CD } 44^{\text {hi }}} \mathrm{CD} 45 \mathrm{RB}^{\mathrm{lo}}$ memory T cells of colon from vehicle- or agonist-treated mice. a Increased GPR15 $\left(\mathrm{GFP}^{+}\right)$memory CD4 ${ }^{+} \mathrm{T}$ cells in colon and small intestinal lamina propria with I3C. Mean + SEM, $n=11$ corn oil, $n=11$ I3C. Combined results of three experiments. b Increased GPR15 in colon, PLN, and spleen with $1 \mu \mathrm{g} / \mathrm{kg}$ doses of TCDD. $n=4$ DMSO, $n=5$ TCDD. c Increased GPR15 expression in intestinal and lymphoid tissues with $10 \mu \mathrm{g} / \mathrm{kg}$ doses of TCDD treatment. $n=9$, except for spleen and PP, where $n=6$. Combined results of three studies. For a-c, two-way ANOVA with Bonferroni posttest; ${ }^{*} P<0.05,{ }^{* * *} P<0.001,{ }^{* * * *} P<0.0001 ;$ PLN peripheral lymph nodes, MLN mesenteric lymph nodes, PP Peyer's patches, SI small intestine.

AHR-mediated GPR15 expression. While our data alludes to synergistic effects of GATA3 and AHR co-expression in promoting GPR15 expression, it does not address the requirement of GATA3 for AHR-mediated GPR15 expression. Mutation of GATA3 binding site in the GPR15 3'enhancer or co-expression of a GATA3-mutant incapable of transcriptional transactivation would likely provide insights into the relative contribution of AHR and GATA3 (interdependent vs. mutually exclusive) in promoting GPR15 expression and merits further consideration.

Our in vitro studies using activated human PBMCs, sorted effector/memory $\mathrm{CD}^{+} \mathrm{T}$ cells, in vitro polarized human Th2 and Treg cells revealed that GPR15 expression is increased in response to AHR agonists and decreased by AHR antagonist (CH223191). These results are supported by recent reports that show GPR15 expression was increased by the AHR agonist, FICZ and decreased by AHR antagonist $(\mathrm{CH} 223191)$ in human Th17 cultures and iTreg cultures. $^{23}$ In the context of GPR15 expression in T cell subsets, the intricate balance between the levels and activity of transcription factors such as GATA3, Foxp3, RORyt, AHR likely activate or suppress GPR15 expression and warrants future investigation.

In the AHR knockout mice, GPR15 expression was greatly reduced in the colon as well as the small intestine, where GPR15 is also expressed but to a much lesser extent. With AHR absence, GPR15 expression decreased on $\mathrm{CD}^{+}{ }^{+} \mathrm{T}$ cells that are CD25-high, indicative of Foxp3 ${ }^{+}$Tregs, as well as effector/memory $\mathrm{CD}^{+}$ $T$ cells that are CD25-low. This expression pattern suggests that the AHR is important for maintaining GPR15 expression on both Tregs and effector/memory $\mathrm{CD} 4^{+} \mathrm{T}$ cells. Tregs express the AHR, and it has been demonstrated that AHR activation by TCDD leads to the development of $\mathrm{CD}_{2} 5^{+} \mathrm{CD}^{+} \mathrm{T}$ cells with regulatory activity. ${ }^{24}$ Absence of the AHR, however, did not significantly reduce the number of total $\mathrm{CD} 25^{+} \mathrm{CD} 4^{+} \mathrm{T}$ cells per se. These results imply a more prominent effect of the AHR on GPR15 expression than on the differentiation of $\mathrm{CD} 25^{+} \mathrm{CD} 4^{+} \mathrm{T}$ cells. Furthermore, in support of our results that AHR regulates GPR15 expression on $\mathrm{CD}^{+}{ }^{+}$Tregs, AHR-deficient Tregs showed decreased expression of GPR $15 .{ }^{25}$ In mixed bone marrow chimeric mice, AHR deficiency also significantly reduced GPR15 expression on effector/memory $\mathrm{CD}^{+}{ }^{+} \mathrm{T}$ cells and $\mathrm{CD} 4^{+}$Tregs of both the small intestine and colon. Decreased GPR15 expression in $\mathrm{Ahr}^{-/-}$mice did not correlate with fewer effector/ memory $\mathrm{CD}^{+}{ }^{+} \mathrm{T}$ cells or Tregs in the colon, but intriguingly with more of these cell populations in the small intestine. Considering that colon $\mathrm{CD}^{+}$ T cells also present integrin a4 $\beta 7$, decreased GPR15 expression on colon $\mathrm{CD}^{+}{ }^{+} \mathrm{T}$ cells due to AHR deficiency could conceivably enable their recruitment to the small intestine. In vivo exposure to high levels of TCDD appear to also increase a4 $\beta 7$ but not CCR9 expression on colon $\mathrm{CD}^{+}{ }^{+} \mathrm{T}$ cells. Additional studies are required to demonstrate the effect of the AHR on small intestinal homing of $\mathrm{CD}^{+} \mathrm{T}$ cells. These results highlight the importance of the AHR in maintaining GPR15 expression, and they indicate a potential function of the AHR in balancing lymphocyte trafficking to the upper and lower intestinal tract.

In addition to the well-known dioxins and environmental pollutants, the AHR binds to an array of natural agonists that are derived from dietary flavonoids and indoles, and tryptophan metabolites produced by microbiota as well as host cells. ${ }^{10-12}$ Recently, it was shown that the receptor also binds and mediates the metabolic degradation of phenazine-containing bacterial virulence factors. ${ }^{26}$ In addition, phenazine-producing bacteria can be found in plants, animal hosts, and the normal human microbiota, such as the Nocardia species that exist in the oral cavity and Methanocercina mazei in the digestive tract. ${ }^{27}$ We showed that GPR15 expression can be induced in vivo or in cell culture by AHR 
agonists, including the dietary compound I3C, natural agonist FICZ, bacterial virulent factors 1-hydroxphenazine and pyocyanin, as well as TCDD. The various known sources of AHR agonists are consistent with the insignificant impact on GPR15 expression by microbiotadeficiency in germ-free mice, and also suggest that GPR15 can be upregulated by AHR agonists with potential for intestinal exposure. We also report that the AHR mediates GPR15 expression on CD4 ${ }^{+}$ $T$ cells in vivo following agonist exposure and augments the guthoming capability of $\mathrm{GPR} 5^{+}$effector/memory $\mathrm{CD}^{+} \mathrm{T}$ cells, especially to the colon. With repeated agonist administration, GPR15 was upregulated on intestinal CD4 ${ }^{+} \mathrm{T}$ cells, most notably in the colon, the site of high GPR15L expression. We did not observe in vivo GPR15 induction with one dose of TCDD at shorter time points (not shown) but with prolonged dosing. Cumulative treatment at a higher concentration of TCDD $(10 \mu \mathrm{g} / \mathrm{kg})$ induced GPR15 on $\mathrm{CD}^{+} \mathrm{T}$ cells at all lymphoid and intestinal tissues examined; in the colon, over $50 \%$ of effector/memory $\mathrm{CD}^{+}{ }^{+} \mathrm{T}$ cells expressed the receptor, including a distinct subpopulation of GPR15bright cells with noted low expression of CD25. Whether this population carries particular effector function and biological impact remains to be determined.

Our findings are consistent with the recent report by Xiong et al., that AHR promotes GPR15 expression on human and mouse $\mathrm{CD}^{+}{ }^{+}$T cells. ${ }^{19}$ Though unrelated and beyond the scope of the current study, Xiong et al., reported GPR15 expression by human colon Tregs (from "normal tissue of patients with ulcerative colitis"), which is contrary to our previous publication. ${ }^{2}$ However, consistent with our results, Adamczyk et al., reported very low level of GPR15 expression on Tregs in the 'inflamed' areas of the colon of ulcerative colitis patients and in the colon of healthy subjects in contrast to higher GPR15 expression on Tregs isolated from 'non-inflamed' colon region in ulcerative colitis patients. ${ }^{28}$ This alludes to the possibility of variable GPR15 expression pattern in the colon of ulcerative colitis patients depending on disease activity and could reconcile the observed differences in GPR15 expression on colonic Tregs in our previous study and that of Xiong et al., and needs further investigation.

The AHR can mediate both immune regulatory and pathological effector functions, the determinant of which is a proposed combination of agonist stimuli and immune milieu that either dampens an immune response to maintain homeostasis or heightens an effector response against toxins and pathogens. ${ }^{29}$ A large body of evidence supports a protective role of the AHR in gut inflammation, evident by the amelioration of experimental colitis by TCDD-induced colonic Tregs. ${ }^{18,30,31}$ On the other hand, Th17 cells also express the AHR, and FICZ-enhanced Th17 differentiation can exacerbate disease in the murine model of experimental autoimmune encephalomyelitis. ${ }^{32}$ We have previously shown that GPR15 mediates experimental colitis and is expressed on colon lamina propria effector $\mathrm{CD}^{+} \mathrm{T}$ cells of ulcerative colitis patients. In this investigation, we demonstrate a novel role for the AHR in regulating GPR15 expression. Consistent with a pro-inflammatory function, AHR-mediated GPR15 expression on effector/memory $\mathrm{CD}^{+}{ }^{+} \mathrm{T}$ cells may serve to respond to toxic agents or pathogens through increased lymphocyte trafficking to the colon. In sum, the relative contribution of AHR signaling and transcription factors capable of binding to the GPR15 $3^{\prime}$ enhancer, in part, governed by factors in the tissue microenvironment in health or disease, likely regulates GPR15 expression. These are important considerations for future studies and for evaluating the therapeutic modulation of the AHR-GPR15 axis.

\section{METHODS}

GPR15 promoter and enhancer cloning

Genomic DNA was isolated from the GPR15-expressing human lymphoblastoid cell line GM12878 (American Type Culture Collection, Manassas, VA) and used as PCR DNA template for both the GPR15 promoter and wildtype enhancer. A $\sim 1$ kilobase region of the GPR15 promoter was PCR-amplified using oligonucleotides promoter- $\mathrm{F}$ and promoter-R (Supplementary Table 1), which contain the Kpnl or Xhol restriction sites incorporated at the $5^{\prime}$ ends of the sense and antisense strands, respectively. This GPR15 promoter was cloned into the promoterless pGL4.12 [luc2CP] firefly luciferase reporter vector (Promega) by standard enzyme restriction and ligation. The resulting vector was sequence-verified and used to subclone the enhancer containing wildtype or mutant XRE.

The wildtype enhancer was PCR-amplified from GM12878 genomic DNA using the primer pair enhancer-F and enhancer-R that contain BamHI and Sall cleavage sites. The enzyme-restricted $\mathrm{PCR}$ product was ligated $3^{\prime}$ of the luciferase gene to generate the wildtype enhancer vector. A second wildtype enhancer construct was similarly generated using primers enhancer2-F and enhancer $2-R$ to clone the enhancer sequence in the reverse order. Transient transfection of HEK293 cells plus AHR agonist treatment indicated that both wildtype enhancer constructs were similarly active, so we continued the remaining expression studies using the second vector.

Mutations in the XRE were generated by primer extension using primers (Supplementary Table 1) that introduced a Kpnl site into the first XRE $\left(\mathrm{XRE}_{1}\right)$ and EcoRI into the second $\left(\mathrm{XRE}_{2}\right)$, simultaneously resulting in point mutations and enabled screening of $E$. coli transformants by restriction digestion. To construct mutant $\mathrm{XRE}_{1}$, the two PCR products of oligo pairs enhancer2-R with enh2m1-F and enhancer2-F with enh2m1-R were combined and used as templates to generate a $563 \mathrm{bp}$ PCR product of primers enhancer2- $\mathrm{F}$ and enhancer2-R, which was then subcloned to replace the wildtype enhancer. The mutant $X R E_{2}$ vector was similarly generated using the two primer pairs enhancer2- $F$ with enh2m2-R and enhancer2- $R$ with enh2m2-F. Mutant clones were screened by enzyme digestion that included Kpnl (for $\mathrm{XRE}_{1}$ ) and EcoR1 (for $\mathrm{XRE}_{2}$ ). To generate a construct that contains both $X_{R E}$ and $X R E_{2}$ mutants, the secondround PCR products of both mutant XRE were pooled at 1:1 ratio and used as templates for amplification with enhancer2- $F$ and enhancer2-R. All selected mutant clones were confirmed by DNA sequencing (using the $P C R$ primers) with a 2-4-fold coverage (MCLAB, South San Francisco, CA).

Transient transfection

Human HEK293 cells were seeded into 96 -well plates at 10,000 cells per well and incubated overnight. Using Lipofectamine ${ }^{\circledR} 2000$ Transfection Reagent (Thermo Fisher Scientific, Grand Island, NY), cells were transiently co-transfected with three plasmids: (1) the GPR 15 promoter-driven luciferase reporter with WT-XRE or mutant$X R E 3^{\prime}$ enhancer, (2) human AHR expression plasmid pCMV-AHR, and (3) renilla luciferase transfection control plasmid pRL-TK (Promega). For some experiments, cells were transfected with human GATA3 (gift from Arne S. Schaefer, Addgene plasmid 115271) and human Foxp3 (gift from Stefan Koch; Addgene 153147) expression plasmids as well. After $24 \mathrm{~h}$ of transfection, cells were treated with AHR agonists or AHR antagonist (CH223191, SIGMA) and incubated overnight. The PBS-washed cells were lysed by a cycle of freeze-thaw and lysis buffer, and firefly and renilla luciferase activities assessed using the DualLuciferase Reporter Assay System (Promega, Madison, WI).

GPR15 induction in human PBMC and $\mathrm{CD} 4^{+} \mathrm{T}$ cells

De-identified blood obtained from healthy donors (Stanford Blood Center, Stanford, CA) was fractioned by a standard centrifugation protocol using a Ficoll-Paque ${ }^{\circledR}$ PLUS gradient (GE Healthcare Biosciences, Pittsburgh, PA). The buffy coat layer was isolated and washed in HBSS containing $2 \%$ BCS.

Cells were seeded into 24 -well tissue-culture-treated plates at $1 \times 10^{6}$ cells per well and stimulated with plate bound anti-CD3 (clone HIT3a) and soluble anti-CD28 (clone CD28.2, both at $1 \mu \mathrm{g}$ / $\mathrm{mL}$ and from Thermo Fisher Scientific) for 18-24 h. Cells were 
treated with AHR antagonist $(\mathrm{CH} 223191)$ or agonist compounds pyocyanin, 1-hydro-phenazine, FICZ, TCDD, or DMSO vehicle for 24-48 h. Cells were harvested for gene expression at $24 \mathrm{~h}$ and flow cytometry analysis at $24-48 \mathrm{~h}$. For sorting of $\mathrm{CD}^{+}$ $\mathrm{CD}^{2} 5 \mathrm{RO}^{+}$and $\mathrm{RO}^{-} \mathrm{T}$ cells, freshly isolated human PBMC was stained with CD3-FITC, CD4-BV605, and CD45RO-APC-Cy7 (Biolegend, San Diego, CA) and acquired using FACS Aria cell sorter and FACS Diva software (BD Biosciences, San Jose, CA). Sorted cells were treated with AHR ligands for $48 \mathrm{~h}$ and analyzed as described above.

Mice

All animal studies were approved by the Institutional Animal Care and Use Committees of Stanford University. C57BL/6 mice (6 weeks old) were purchased from Jackson Laboratory and used as recipients in bone marrow chimeras. Gpr15 grp/gfp mice, as previously described, ${ }^{2}$ were crossed with B6.SJL (Jackson Laboratory) to obtain congenic CD45.1 $1^{+}$progeny, which were subsequently bred with $\mathrm{Ahr}^{-1-}$ (CD45.2). Gender- and age-matched progeny, Gpr15 $5^{g f p /,+} A h r^{+/+}$, and $\mathrm{Gpr}_{1} 5^{\mathrm{gfp} /,+} \mathrm{Ahr}^{-/-}$littermates were used in bone marrow chimeras and phenotyping studies. Gpr15 $5^{g f p /+}$ mice used in the in vivo GPR15 induction studies were obtained from crossing Gpr15 $15^{g f / g f p}$ to C57BL/6 mice. Both male and female mice between 8-16 weeks old were used in separate studies.

Bone marrow chimeras

Femur bone marrow of congenic GPR15-sufficient Gpr15 gfp/,+ $\mathrm{Ahr}^{+/+}$(CD45.1) and Gpr15 gfpl, $A$ Ahr ${ }^{-1-}$ (CD45.1, CD45.2) were transferred i.v. in equal proportion $\left(2.5 \times 10^{6}\right.$ cells of each) into lethally irradiated (900 gy) C57BL/6 mice (CD45.2). The mice were given antibiotic-containing chow for 2 weeks. After 6-10 weeks post injection, tissues were harvested, and single-cell suspensions prepared for flow cytometric analysis. For each tissue, the percentage of GPR15-expressing effector/memory (CD44 ${ }^{\text {hi }}$, CD45RB $\left.{ }^{\mathrm{lo}}\right)$ or naive $\left(\mathrm{CD} 44^{\mathrm{lo}}, \mathrm{CD} 4 \mathrm{RB}^{\mathrm{hi}}\right) \mathrm{CD}^{+} \mathrm{T}$ cells were determined. In addition, the $\mathrm{Ahr}^{-/-}(\mathrm{CD} 45.1, \mathrm{CD} 45.2)$ to $\mathrm{Ahr}^{+/+}$ (CD45.1) ratio of these $\mathrm{CD}^{+} \mathrm{T}$ cell subsets were calculated and normalized to that of the spleen to control for differences in engraftment of donor bone marrow between individual mice.

In separate experiments, femur bone marrow of congenic GPR15-sufficient $\mathrm{Ahr}^{+/+}$(CD45.1) and $\mathrm{Ahr}^{-/-}$(CD45.2) were transferred intravenously into irradiated B6.SJL.F1 mice (CD45.1, CD45.2) (Jackson Laboratory) as outlined above. After 15 weeks (longer time-point due to COVID-19-related lab shut down in early 2020) post injection, tissues were harvested, stained, and analyzed by flow cytometry. An anti-mouse GPR15-PE antibody (Biolegend) was used to detect GPR15.

In vivo induction of GPR15

A suspension of Indole-3-carbinol (I3C; Sigma-Aldrich, St. Louis, MI) was prepared in corn oil $(100 \mathrm{mg} / \mathrm{mL})$. AHR wildtype, Gpr $15^{g f p /,+}$ mice were orally gavaged with $600 \mathrm{mg} / \mathrm{kg}(\approx 150 \mu \mathrm{L}$ per $20 \mathrm{~g}$ mouse) once daily, five times a week for 3 weeks. In separate studies, 2,3,7,8-tetrachlorodibenzo-p-dioxin (TCDD; purchased from AccuStandard, New Haven, CT) was dissolved in DMSO to a stock concentration of $5 \mu \mathrm{g} / \mathrm{mL}$. Gpr $15^{g f p /,+}$ mice were i.p. injected with $1 \mu \mathrm{g} / \mathrm{kg}$ or $10 \mu \mathrm{g} / \mathrm{kg}$ (or $40 \mu \mathrm{L}$ per $20 \mathrm{~g}$ mouse) three times a week (every other day) for 3 weeks. Expression of GPR15 on tissue lymphocytes was assessed by flow cytometry. In separate experiments, GPR15-sufficient $\mathrm{Ahr}^{+/+}$(CD45.1) and $\mathrm{Ahr}^{-1-}(\mathrm{CD} 45.2)$ treated with DMSO or $10 \mu \mathrm{g} / \mathrm{kg}$ of TCDD and expression of GPR15 on tissue lymphocytes was analyzed using GPR15-PE antibody (Biolegend).

\section{ACKNOWLEDGEMENTS}

We thank Yi Ta Wei and Yujun Yang for technical assistance with the animal work and cloning studies. This work was supported by NIH grant R01 DK101119 to A.H., NIH grant R37 Al047822 and a Merit Award from the Department of Veterans Affairs to E.C.B. This work was also supported by the Ann and Bill Swindells Charitable Trust, and Leslie and Douglas Ballinger.

\section{AUTHOR CONTRIBUTIONS}

G.S. and L.P.N. designed, performed the studies, analyzed data, and wrote the manuscript. H.K. and Y.H. designed, performed in vivo studies, and analyzed data. J.P. performed the comparative sequence analysis, identified the AHR binding sites that led to the project's inception, contributed to the design of the cloning and reporter studies, provided advice, and edited the manuscript. T.T.D. designed the cloning studies, which were performed by A.P., L.V.M. and L.P.N. H.S. performed some animal studies, A.J. and D.M. performed some in vitro and in vivo studies, and B.L. provided technical assistance. E.C.B. provided scientific input and guidance. AH supervised the studies, provided guidance, analyzed data, and wrote the manuscript.

\section{ADDITIONAL INFORMATION}

Supplementary information The online version contains supplementary material available at https://doi.org/10.1038/s41385-021-00390-x.

Competing interests: The authors declare no competing interests.

Publisher's note Springer Nature remains neutral with regard to jurisdictional claims in published maps and institutional affiliations.

\section{REFERENCES}

1. Kim, S. V. et al. GPR15-mediated homing controls immune homeostasis in the large intestine mucosa. Science 340, 1456-1459 (2013).

2. Nguyen, L. P. et al. Role and species-specific expression of colon T cell homing receptor GPR15 in colitis. Nat. Immunol. 16, 207-213 (2015).

3. Suply $T$. et al. A natural ligand for the orphan receptor GPR15 modulates lymphocyte recruitment to epithelia. Sci. Signal. 10, eaal0180 (2017).

4. Ocón, B. et al. A mucosal and cutaneous chemokine ligand for the lymphocyte chemoattractant receptor GPR15. Front. Immunol. 8, 1111 (2017).

5. Kiene, M. et al. Toll-like receptor 3 signalling up-regulates expression of the HIV co-receptor G-protein coupled receptor 15 on human CD4+ T cells. PLOS ONE 9, e88195 (2014)

6. Konkel, J. E. et al. Transforming growth factor- $\beta$ signaling in regulatory $t$ cells controls $\mathrm{t}$ helper-17 cells and tissue-specific immune responses. Immunity 46, 660-674 (2017).

7. Dignass, A. U. \& Podolsky, D. K. Cytokine modulation of intestinal epithelial cell restitution: central role of transforming growth factor beta. Gastroenterology 105, 1323-1332 (1993).

8. Gu, Y. Z., Hogenesch, J. B. \& Bradfield, C. A. The PAS superfamily: sensors of environmental and developmental signals. Annu. Rev. Pharm. Toxicol. 40, 519-561 (2000).

9. Kewley, R. J., Whitelaw, M. L. \& Chapman-Smith, A. The mammalian basic helixloop-helix/PAS family of transcriptional regulators. Int. J. Biochem. Cell Biol. 36, 189-204 (2004).

10. Denison, M. S., Soshilov, A. A., He, G., DeGroot, D. E. \& Zhao, B. Exactly the same but different: promiscuity and diversity in the molecular mechanisms of action of the aryl hydrocarbon (dioxin) receptor. Toxicol. Sci. 124, 1-22 (2011).

11. Nguyen, L. P. \& Bradfield, C. A. The search for endogenous activators of the aryl hydrocarbon receptor. Chem. Res. Toxicol. 21, 102-116 (2008).

12. Barroso, A., Mahler, J. V., Fonseca-Castro, P. H., Quintana, F. J. The aryl hydrocarbon receptor and the gut-brain axis. Cell Mol. Immunol. 18, 259-268 (2021).

13. Kiss, E. A. et al. Natural aryl hydrocarbon receptor ligands control organogenesis of intestinal lymphoid follicles. Science 334, 1561-1565 (2011).

14. Lee, J. S. et al. AHR drives the development of gut ILC22 cells and postnatal lymphoid tissues via pathways dependent on and independent of Notch. Nat. Immunol. 13, 144-151 (2011).

15. Qiu, J. et al. The aryl hydrocarbon receptor regulates gut immunity through modulation of innate lymphoid cells. Immunity 36, 92-104 (2012).

16. Esser, C. \& Rannug, A. The aryl hydrocarbon receptor in barrier organ physiology, immunology, and toxicology. Pharm. Rev. 67, 259-279 (2015).

17. Rothhammer, V. \& Quintana, F. J. The aryl hydrocarbon receptor: an environmental sensor integrating immune responses in health and disease. Nat. Rev. Immunol. 19, 184-197 (2019).

18. Lamas, B., Natividad, J. M. \& Sokol, H. Aryl hydrocarbon receptor and intestinal immunity. Mucosal Immunol. 11, 1024-1038 (2018). 
19. Xiong, L. et al. Ahr-Foxp3-RORyt axis controls gut homing of CD4. Sci. Immunol. 5, eaaz7277 (2020).

20. Bjeldanes, L. F., Kim, J. Y., Grose, K. R., Bartholomew, J. C. \& Bradfield, C. A. Aromatic hydrocarbon responsiveness-receptor agonists generated from indole3-carbinol in vitro and in vivo: comparisons with 2,3,7,8-tetrachlorodibenzo-pdioxin. Proc. Natl Acad. Sci. USA 88, 9543-9547 (1991).

21. Wheeler, J. L., Martin, K. C., Resseguie, E. \& Lawrence, B. P. Differential consequences of two distinct $A h R$ ligands on innate and adaptive immune responses to influenza A virus. Toxicol. Sci. 137, 324-334 (2014).

22. Veldhoen, M., Hirota, K., Christensen, J., O'Garra, A. \& Stockinger, B. Natural agonists for aryl hydrocarbon receptor in culture medium are essential for optimal differentiation of Th17 T cells. J. Exp. Med. 206, 43-49 (2009).

23. McAleer, J. P., Fan, J., Roar, B., Primerano, D. A. \& Denvir, J. Cytokine regulation in human CD4 T Cells by the aryl hydrocarbon receptor and Gq-coupled receptors. Sci. Rep. 8, 10954 (2018).

24. Funatake, C. J., Marshall, N. B., Steppan, L. B., Mourich, D. V. \& Kerkvliet, N. I. Cutting edge: activation of the aryl hydrocarbon receptor by 2,3,7,8-tetrachlorodibenzo-p-dioxin generates a population of $\mathrm{CD} 4+\mathrm{CD} 25+$ cells with characteristics of regulatory T cells. J. Immunol. 175, 4184-4188 (2005).
25. Ye, J. et al. The aryl hydrocarbon receptor preferentially marks and promotes gut regulatory T cells. Cell Rep. 21, 2277-2290 (2017).

26. Moura-Alves, $P$. et al. AhR sensing of bacterial pigments regulates antibacterial defence. Nature 512, 387-392 (2014).

27. Pierson, L. S. 3rd. \& Pierson, E. A. Metabolism and function of phenazines in bacteria: impacts on the behavior of bacteria in the environment and biotechnological processes. Appl. Microbiol. Biotechnol. 86, 1659-1670 (2010).

28. Adamczyk, A. et al. Differential expression of GPR15 on T cells during ulcerative colitis. JCI Insight. 2, e90585 (2017).

29. Julliard, W., Fechner, J. H. \& Mezrich, J. D. The aryl hydrocarbon receptor meets immunology: friend or foe? A little of both. Front Immunol. 5, 458 (2014).

30. Benson, J. M. \& Shepherd, D. M. Aryl hydrocarbon receptor activation by TCDD reduces inflammation associated with Crohn's disease. Toxicol. Sci. 120, 68-78 (2011).

31. Singh, N. P. et al. Activation of aryl hydrocarbon receptor (AhR) leads to reciprocal epigenetic regulation of FoxP3 and IL-17 expression and amelioration of experimental colitis. PLoS ONE 6, e23522 (2011).

32. Veldhoen, M. et al. The aryl hydrocarbon receptor links TH17-cell-mediated autoimmunity to environmental toxins. Nature 453, 106-109 (2008). 